\title{
Randomized Controlled Trial to Compare the Safety and Efficacy of Tamsulosin, Solifenacin, and Combination of Both in Treatment of Double-J Stent-Related Lower Urinary Symptoms
}

\author{
Essam Shalaby, ${ }^{1,2}$ Abul-fotouh Ahmed, ${ }^{3,4}$ Aref Maarouf,, ${ }^{5,6}$ Iman Yahia, ${ }^{1,7}$ \\ Mohamed Ali, 1 and Ammar Ghobish ${ }^{1,7}$ \\ ${ }^{1}$ Department of Urology, Suez Canal University, P.O. Box 224, Ismailia 41522, Egypt \\ ${ }^{2}$ Department of Urology, King Khalid Hospital, P.O. Box 1985, Al-Kharj 11942, Saudi Arabia \\ ${ }^{3}$ Department of Urology, Al-Azhar University, Nasr City, Cairo 11884, Egypt \\ ${ }^{4}$ Department of Urology, Salman Bin Abdul-Aziz University, P.O. Box 173, Al-Kharj 11942, Saudi Arabia \\ ${ }^{5}$ Department of Urology, Faculty of Medicine, Zagazig University, P.O. Box 203, Zagazig 44519, Egypt \\ ${ }^{6}$ Department of Urology, King Abdallah Hospital, P.O. Box 60, Bisha 61922, Saudi Arabia \\ ${ }^{7}$ Department of Urology, Insurance Hospital, Ismailia 41528, Egypt
}

Correspondence should be addressed to Abul-fotouh Ahmed; abulfotouhahmed@yahoo.com

Received 2 June 2013; Accepted 7 September 2013

Academic Editor: James A. Brown

Copyright (c) 2013 Essam Shalaby et al. This is an open access article distributed under the Creative Commons Attribution License, which permits unrestricted use, distribution, and reproduction in any medium, provided the original work is properly cited.

Purpose. We evaluated the effectiveness and safety of tamsulosin, solifenacin, and combination of both in reducing double-J stentrelated lower urinary symptoms. Materials and Methods. A total of 338 patients with double-J ureteral stenting were randomly divided, postoperatively, into 4 groups. In group I $(n=84)$, no treatment was given (control group), group II $(n=85)$ received tamsulosin $0.4 \mathrm{mg}$ daily, group III $(n=84)$ received solifenacin $10 \mathrm{mg}$ daily, and group IV $(n=85)$ received a combination of both medications. Before insertion and 2 weeks after, all patients completed the International Prostate Symptom Score (IPSS), quality of life component of the IPSS (IPSS/Qol), Overactive Bladder Questionnaire (OAB-q), and Visual Analogue Pain Scale (VAPS) questionnaire. Results. The demographics and preoperative questionnaires scores of all groups were comparable. There were statistically significant differences in all scores in favour of groups II, III, and IV as compared to control group ( $P$ value $<$ 0.005). Group IV showed statistically significant differences in total IPSS, QoL score, and OAB-q score as compared to groups II and III ( $P$ value $<0.001)$. Conclusions. Combined therapy of tamsulosin and solifenacin significantly alleviated lower urinary symptoms associated with double-J stents as compared to either medication alone.

\section{Introduction}

The double-J stents are common tools and integral part used in endourologic practices. Double-J stents play a major role in a wide range of situations to prevent or to relieve ureteral obstruction $[1,2]$. Despite the usefulness of double-J stent, some of the patients might encounter stent-related morbidities such as urinary tract infection (UTI), lower urinary tract symptoms (LUTS), stent-related body pain, and hematuria. These symptoms represent a prevalent problem with considerable effects on the quality of life, substantial general health, work performance, and sexual matters in both genders [3-5]. The pathophysiology of stent-related symptoms remains unclear. However, the pain and LUTS caused by stent placement has been attributed to lower ureter and bladder spasm due to local irritation of the stent [6]. Some previous studies indicated oral agents such as alpha1-blockers and antimuscarinic agents to relieve LUTS associated with double-J ureteral stents [7-10].

Tamsulosin acts as a selective inhibitor of $\alpha$-1a/ldmediated contraction of the smooth muscles in distal ureter, bladder trigone, and bladder neck [11]. It is thought that relaxing these smooth muscles decreases bladder outlet resistance and voiding pressure, with beneficial effect on stent 
related LUTS $[7,10]$. Solifenacin acts as a muscarinic receptor antagonist used for treatment of patients with overactive bladder $(\mathrm{OAB})[12,13]$ and might be effective as well for stentrelated symptoms [8-10].

In this prospective study we evaluated the effectiveness and safety of alpha-1-blocker (tamsulosin), antimuscarinic (solifenacin), and combination of both medications in reducing double-J stent-related LUTS, using the International Prostate Symptom Score (IPSSs), quality of life component of the IPSS (IPSS/Qol), Overactive Bladder Questionnaire (OAB-q), and Visual Analogue Pain Scale (VAPS) questionnaire.

\section{Patients and Methods}

2.1. Study Population and Design. This multicentre, prospective, randomized, and comparative study was carried out between November 2009 and March 2013. Patients were selected among those underwent retrograde double-J ureteral stent placement, before ESWL or following ureterorenoscopy (URS), ureterolithotomy, percutaneous nephrolithotomy (PCNL), and endoscopic endopyelotomy. The study was carried out by five urologists at four institutions (King Khalid Hospitals "KKH," Salman Bin Abdul-Aziz University Hospital "SAUH" (Al-Kharj, KSA), King Abdullah Hospital "KAH" (Bisha, KSA), and Ismailia Insurance Hospital "IIH" (Ismailia, Egypt)). Patients $\geq 18$ years of age with unilateral double-J ureteral stent who agreed to random allocation of treatment were eligible for enrolment. Patients were excluded if they met any of the following criteria: (1) age less than 18 years, (2) pregnant women, (3) history of previous ureteral stenting, (4) bilateral stents, (5) long-term stenting (on regular exchange), (6) bladder pathology, (7) benign prostatic hyperplasia, (8) overactive bladder, (9) urinary tract infection, or (9) previous use of selective alpha-1-blocker and/or antimuscarinic agent.

2.2. Study Procedure. A total of 338 patients were enrolled in study (172 patients from KKH and SAUH, 102 patients from $\mathrm{KAH}$, and 64 patients from $\mathrm{IIH})$. A computer randomization program (http://www.randomization.com/) was used to allocate patients into four groups. We used randomization in blocks of four, and each center had its own list to keep the groups closely balanced. Patients in group I $(n=84)$ received no treatment (control group) (C group), patients in group II $(n=85)$ received a daily oral dose of tamsulosin $0.4 \mathrm{mg}$ ( T group), patients in group III $(n=84)$ received solifenacin succinate $10 \mathrm{mg}$ (S group), and patients in group IV $(n=85)$ received a combination of the two medications once daily (T/S group). Routine preoperative evaluation was done for the planned operative procedures. A polyurethane ureteral stents were used in all patients. The length and calibre were adjusted for each patient. Only the coiled distal end was present in the bladder without any part of distal shaft. Routinely, X-ray abdominal film was done for all patients before home discharge to confirm the proper stent positioning. The protocol and all study procedures were conducted in conformity with the ethical guidelines of the Declaration of Helsinki of 1975. The study protocol was approved by the ethics committee of the participating hospitals, and all patients enrolled in this study provided written informed consent.

2.3. Patients Assessment and Outcome Measurements. Before and two weeks after stent insertion, all patients completed written International Prostate Symptom Score/quality of life component of IPSS (IPSS/QoL), Overactive Bladder Questionnaire (OAB-q), and Visual Analogue Pain Scale (VAPS) questionnaires. The IPSS was divided into the total score, voiding symptom score, and storage symptom score, and each one was compared. Visual Analogue Pain Scale graded from 1 (minimal or no symptoms) to 10 (symptoms of maximal severity).

2.4. Statistical Analysis. Using SPSS software ver. 18.0 (SPSS Inc., Chicago, IL, USA), statistical analysis was performed. Chi-square test, ANOVA, and one-way repeated-measures ANOVA were used for comparisons between the 4 groups. When comparing postinsertion to preinsertion scores, a statistically significant difference reflects poor control of stent related symptoms. The power used was 0.80 , and the level of significance was $5 \%$.

\section{Results}

A total of 338 patients were enrolled in the study, and 327 patients completed the study (3 patients from group I, 3 patients from group II, 4 patients from group III, and 1 patient from group IV dropped out). Group I (81 patients) consisted of 50 men and 31 women (mean age: $44.0 \pm 15.2$ years), group II (82 patients) consisted of 55 men and 27 women (mean age: $41.3 \pm 17.1$ years), group III (80 patients) consisted of 57 men and 23 women (mean age: $40.5 \pm 18.6$ years), and group IV (84 patients) consisted of 58 men and 26 women (mean age: $43.6 \pm 17.6$ years). The main indication of ureteral double-J stent placement was URS and ureterolithotomy. All patients completed the necessary questionnaires. There were no statistically significant differences between groups regarding patient's demographics, treatment indications, and preoperative questionnaires scores (Tables 1 and 2).

Therapies were well tolerated, and no patients discontinued the treatments because of side effects. When comparing the pre- and postinsertion scores, we found that there was a statistically significant difference in all evaluated scores in the control group, and this represents poor control of stent related symptoms. In single therapy groups ( $\mathrm{T}$ and $\mathrm{S}$ groups) each drug controlled voiding symptoms $(P=0.698$ and 0.411$)$ but not other stent related symptoms. However, in combination therapy (T/S group) the differences were nonsignificant in all evaluated scores indicating maximum symptom control.

When comparing poststenting scores among different groups, there were a statistically significant differences in all scores in favour of groups II, III, and IV as compared to group I $(P$ value $<0.005)$. However when we compared each group by one-way ANOVA at each time point separately, there was no statistically significant difference between groups II and III as regards (total, storage, and voiding) IPSS scores $(P=$ $0.352,0.07$, and 0.513 , resp.), although there was statistically 
TABLE 1: Basic characteristics of studied patients.

\begin{tabular}{lcccc}
\hline Variables & Group I & Group II & Group III & Group IV \\
& C group & T group & S group & T/S group \\
\hline Number of patients & 81 & 82 & 80 & 84 \\
Mean age (years) & $44.0 \pm 15.2$ & $41.3 \pm 17.1$ & $40.5 \pm 18.6$ & $43.6 \pm 17.6$ \\
Sex (male : female) & $1.6: 1$ & $2: 1$ & $2.5: 1$ & $2.2: 1$ \\
Indications of stent placement & & & 59 & 63 \\
$\quad$ URS/ureterolithotomy & 56 & 57 & 07 & 05 \\
PCNL & 07 & 10 & 12 & 13 \\
ESWL & 16 & 14 & 02 & 03 \\
$\quad$ Endopyelotomy & 02 & 01 & 5 & \\
\hline
\end{tabular}

URS: Ureterorenoscopy; PCNL: Percutaneous Nephrolithotomy; ESWL: Extracorporeal Shock Wave Lithotripsy.

TABLE 2: Comparisons of IPSS/QoL, VAP scale, and OAB-q scores in all groups.

\begin{tabular}{|c|c|c|c|c|c|}
\hline & $\begin{array}{l}\text { Group I } \\
\text { C group }\end{array}$ & $\begin{array}{l}\text { Group II } \\
\mathrm{T} \text { group }\end{array}$ & $\begin{array}{l}\text { Group III } \\
\text { S group }\end{array}$ & $\begin{array}{l}\text { Group IV } \\
\mathrm{T} / \mathrm{S} \text { group }\end{array}$ & $P$ value \\
\hline \multicolumn{6}{|c|}{ IPSS (storage symptom score) } \\
\hline Preinsertion & $4.18 \pm 2.48$ & $4.25 \pm 2.54$ & $4.30 \pm 2.65$ & $4.30 \pm 2.41$ & 0.989 \\
\hline 2 weeks after insertion & $8.24 \pm 3.44$ & $7.68 \pm 3.66$ & $6.62 \pm 3.92$ & $4.66 \pm 3.24$ & 0.000 \\
\hline \multicolumn{6}{|c|}{ IPSS (voiding symptom score) } \\
\hline Preinsertion & $4.62 \pm 2.86$ & $4.66 \pm 2.66$ & $4.75 \pm 2.55$ & $4.62 \pm 2.46$ & 0.988 \\
\hline 2 weeks after insertion & $7.22 \pm 2.46$ & $4.82 \pm 2.62$ & $5.10 \pm 2.82$ & $3.82 \pm 2.02$ & 0.000 \\
\hline \multicolumn{6}{|l|}{ IPSS (total score) } \\
\hline Preinsertion & $8.80 \pm 4.42$ & $8.91 \pm 4.26$ & $9.05 \pm 3.87$ & $8.92 \pm 4.12$ & 0.986 \\
\hline 2 weeks after insertion & $15.46 \pm 4.28$ & $12.40 \pm 4.50$ & $11.72 \pm 4.77$ & $8.48 \pm 4.22$ & 0.000 \\
\hline \multicolumn{6}{|l|}{ Qol score } \\
\hline Preinsertion & $1.82 \pm 1.62$ & $1.78 \pm 1.70$ & $1.62 \pm 1.54$ & $1.77 \pm 1.44$ & 0.862 \\
\hline 2 weeks after insertion & $4.12 \pm 1.76$ & $2.80 \pm 1.52$ & $3.36 \pm 1.77$ & $1.88 \pm 1.22$ & 0.000 \\
\hline \multicolumn{6}{|l|}{ OAB-q score } \\
\hline Preinsertion & $8.2 \pm 1.2$ & $8.3 \pm 1.3$ & $8.1 \pm 1.5$ & $8.5 \pm 1.3$ & 0.255 \\
\hline 2 weeks after insertion & $20.53 \pm 2.22$ & $14.93 \pm 1.62$ & $12.42 \pm 1.42$ & $8.44 \pm 1.22$ & 0.000 \\
\hline \multicolumn{6}{|l|}{ VAPS score } \\
\hline Preinsertion & $2.30 \pm 1.46$ & $2.55 \pm 1.84$ & $2.69 \pm 1.46$ & $2.22 \pm 1.27$ & 0.172 \\
\hline 2 weeks after insertion & $5.88 \pm 1.65$ & $3.23 \pm 1.23$ & $4.36 \pm 1.44$ & $2.69 \pm 1.41$ & 0.000 \\
\hline
\end{tabular}

IPSS: International Prostate Symptom Score; QoL: quality of life; OAB-q: Overactive Bladder Questionnaire; VAPS: Visual Analogue Pain Scale.

significant difference in OAB-q and VAPS scores in favour of group II $(P=0.00)$ (Table 3$)$. Group IV patients who received combination therapy showed a statistically significant difference in all scores as compared to monotherapy (II, III) groups $(P$ value $<0.001)$. This confirmed the superiority of combination therapy in overcoming stent-related symptoms as compared to monotherapy.

\section{Discussion}

The results of this prospective, randomized, controlled trial showed that, the combined use of tamsulosin and solifenacin improved the QoL and alleviated LUTS associated with double-J ureteral stents, better than either drug alone and well tolerated.

Stent discomfort is believed to affect over $80 \%$ of patients $[14,15]$. Patients with indwelling stents have been known to complain of a variety of stent-related symptoms, typically: storage, voiding, $\mathrm{OAB}$ symptoms, haematuria, and pain. These symptoms are believed to be unavoidable and associated with reduced health-related quality of life [4].
TABle 3: Comparison between group II and III two weeks post insertion of Double-J stent.

\begin{tabular}{lccc}
\hline & $\begin{array}{c}\text { Group II } \\
\text { T group }\end{array}$ & $\begin{array}{c}\text { Group III } \\
\text { S group }\end{array}$ & $P$ value \\
\hline $\begin{array}{l}\text { IPSS Storage symptom } \\
\text { score }\end{array}$ & $7.68 \pm 3.66$ & $6.62 \pm 3.92$ & 0.07 \\
$\begin{array}{l}\text { IPSS Voiding symptom } \\
\text { score }\end{array}$ & $4.82 \pm 2.62$ & $5.10 \pm 2.82$ & 0.513 \\
IPSS Total score & $12.40 \pm 4.5$ & $11.72 \pm 4.77$ & 0.352 \\
QoL score & $2.80 \pm 1.52$ & $3.36 \pm 1.77$ & 0.013 \\
OAB-q score & $14.93 \pm 1.62$ & $12.42 \pm 1.42$ & 0.000 \\
VAPS score & $3.23 \pm 1.23$ & $4.36 \pm 1.44$ & 0.000 \\
\hline
\end{tabular}

IPSS: International Prostate Symptom Score; QoL: Quality of Life; OAB-q: Overactive Bladder Questionnaire; VAPS: Visual Analogue Pain Scale.

The exact pathophysiology of stent related symptoms remains unknown; it could be related to lower ureteral smooth muscle spasms and local irritation to neuronal-rich 
trigonal mucosa, which contains $\alpha$-1D receptors and bladder instability which give symptoms similar to benign prostatic hyperplasia $[6,16,17]$. To overcome the bothering short term stent related symptoms, some investigators reported that stent length, girth adjustment and avoiding distal end crossing the midline are essential and significantly decrease stent's symptoms [18]. A different design was introduced in the Tail Stent model (Microvasive Urology/Boston Scientific) with proximal 7F pigtail and tapered end $3 \mathrm{~F}$ tail that lie in the bladder. This stent was compared to standard 7F double$J$ stents in a randomized single-blind trial involving 60 patients and showed significant reduction in the stent-related symptoms $[19,20]$. This was in contrary to that reported by Hao et al. [21] and Thomas [6] whom showed no significant effect of length and girth on stent symptoms. Damiano et al. [22] reported that there was no symptoms difference between stent with different size, whereas there was a tendency of small diameter stents to dislodge more often. Chew et al. [23] reported that changing in body position led to movement of distal end within the bladder and induced more trigonal irritation and stent related symptoms.

Lang and associates [24] stated that a possible mechanism of relief of stent-related symptoms could be smooth muscle relaxation of lower ureter and trigone as well as reducing ureteric motility. Wang and his colleagues [25] suggested that relaxation of bladder neck/prostatic smooth muscle, with consequent reduction in voiding pressure and urinary reflux, is other possible mechanisms for control of stent-related symptoms, setting a rationale behind using alpha blockers in overcoming ureteral stent symptoms.

Another mechanism was thought to be related to stent itself which may unmask or exacerbate preexisting subclinical detrusor overactivity causing involuntary bladder contraction, which induces overactive bladder symptoms, setting a rationale behind using antimuscarinic agents to improve stent-related symptoms [26-28]. The symptoms of incontinence and urge incontinence may be explained by stent migration into the proximal urethra which interferes with the urethral sphincter mechanism of continence [28].

The effectiveness of different therapeutic protocols aiming to improve ureteral stent-related symptoms is under investigation. In the present study, we found that both tamsulosin and solifenacin monotherapy poorly controlled ureteral stent related symptoms ( $P$ value 0.000 ). The effectiveness of alpha blockers in controlling double-J stent-related symptoms was reported previously. Wang et al. [7] in a prospective randomized study comparing tamsulosin to placebo in 79 patients using (USSQ) reported that tamsulosin improved stent related urinary symptoms, QoL, and they recommended its routine use. Also Damiano et al. [17] reported that administration of tamsulosin has a positive effect on stentrelated urinary symptoms, QoL, and VAPS, although this study was not double-blinded or placebo-controlled. Also, several studies reported that other alpha-blocker alfuzosin improved stent-related symptoms and quality of life and reduced analgesic demand compared to the placebo group $[29,30]$. However, to our knowledge, no studies compared the effectiveness of different alpha blockers or antimuscrinic agents on stent-related symptoms. Kuyumcuoglu et al. [31] reported in a prospective randomized study that tamsulosin was not different than placebo in controlling stent-related symptoms.

In our study, solifenacin monotherapy poorly controlled stent-related symptoms evidenced by statistically significant differences in the IPSS total score, storage subscore, QoL, $\mathrm{OAB}-\mathrm{q}$, and VAP scores pre- and post-stent insertion. When compared to placebo almost all scores showed statistically significant differences except for storage subscore which indicated that solifenacin was better than placebo, in controlling irritative LUTS. Similarly, Lee et al. [9] reported in a prospective, randomized, and placebo-controlled study that postoperative solifenacin use was effective and well tolerated for the treatment of LUTS, stent-related body pain, and hematuria irrespective of gender in patients undergoing ureteroscopic lithotripsy (URSL) and double-J stent indwelling.

Also, researchers studied the effect of others antimuscrinic agents in reducing the negative symptoms associated with double-J ureteral stent. Norris et al. [32] reported in a prospective, randomized, and double-blinded placebocontrolled study that there were no differences between oxybutynin and placebo in controlling stent-related symptoms. However, they recommended further study on a large number of patients for optimal management of ureteral stent symptoms. Kuyumcuoglu et al. [31] reported that tolterodine SR $4 \mathrm{mg}$ was not different than anti-inflammatory and alpha blocker in controlling stent-related symptoms. In contrast to this data Park et al. [33] in a prospective randomized controlled study reported that tolterodine was significantly able to improve pain and urinary symptom index scores when compared with alfuzosin and placebo.

A limitation of our study was the lack of patient homogeneity (as we included patients with different urologic procedures). However, the indications of double-J stent insertion were statistically similar in the four groups, and our main focus was to compare the efficacy of tamsulosin versus solifenacin versus combined treatment as this has been studied in only few literatures. Our findings showed that combined therapy was better than either tamsulosin or solifenacin monotherapy in reducing stent related symptoms. The superiority of combined tamsulosin and solifenacin therapy was also reported previously by Lim and his colleagues [34] who reported in nonrandomized, retrospective study that combined use of solifenacin and tamsulosin was significantly better than either drug alone in reducing stent related symptoms. However, the small groups of each scale made it difficult to verify the statistical significance, and authors recommended further large-scale, randomized, and prospective study to get more accurate information. In contrast, Lee et al. [35] in their prospective randomized study over 20 patients using a combination of Tamsulosin and tolterodine reported no statistically significant difference when compared to placebo, and also when combination therapy was compared to tamsulosin monotherapy, no beneficial effect was reported. They stated that correct stent positioning and verification of its location were more important than medication for lessening stent-related storage symptoms. 


\section{Conclusions}

Combination therapy of tamsulosin and solifenacin is significantly better than either drug alone in reducing LUTS associated with double-J stents. Combination therapy should be strongly considered for patients who complain of stentrelated symptoms. However, in our opinion, there is need for further studies to compare the effectiveness of combination of different alpha blockers and antimuscrinic agents in order to optimize medical therapy for treatment of symptoms related to stent placement.

\section{References}

[1] B. E. Knudsen, D. T. Beiko, and J. D. Denstedt, "Stenting after ureteroscopy: pros and cons," Urologic Clinics of North America, vol. 31, no. 1, pp. 173-180, 2004.

[2] B. H. Chew, B. E. Knudsen, and J. D. Denstedt, "The use of stents in contemporary urology," Current Opinion in Urology, vol. 14, no. 2, pp. 111-115, 2004.

[3] H. B. Joshi, A. Stainthorpe, F. X. Keeley Jr., R. Macdonagh, and A. G. Timoney, "Indwelling ureteral stents: evaluation of quality of life to aid outcome analysis," Journal of Endourology, vol. 15, no. 2, pp. 151-154, 2001.

[4] H. B. Joshi, N. Newns, A. Stainthorpe, R. P. MacDonagh, F. X. Keeley Jr., and A. G. Timoney, "Ureteral stent symptom questionnaire: development and validation of a multidimensional quality of life measure," Journal of Urology, vol. 169, no. 3, pp. 1060-1064, 2003.

[5] M. C. Sighinolfi, S. Micali, S. de Stefani et al., "Indwelling ureteral stents and sexual health: a prospective, multivariate analysis," Journal of Urology, vol. 178, no. 1, pp. 229-231, 2007.

[6] R. Thomas, "Indwelling ureteral stents: impact of material and shape on patient comfort," Journal of Endourology, vol. 7, no. 2, pp. 137-140, 1993.

[7] C.-J. Wang, S.-W. Huang, and C.-H. Chang, "Effects of specific $\alpha-1 \mathrm{~A} / 1 \mathrm{D}$ blocker on lower urinary tract symptoms due to double-J stent: a prospectively randomized study," Urological Research, vol. 37, no. 3, pp. 147-152, 2009.

[8] M.-S. Choo, J. Z. Lee, J. B. Lee et al., "Efficacy and safety of solifenacin succinate in Korean patients with overactive bladder: a randomised, prospective, double-blind, multicentre study," International Journal of Clinical Practice, vol. 62, no. 11, pp. 16751683, 2008.

[9] Y. J. Lee, K. H. Huang, H. J. Yang, H. C. Chang, J. Chen, and T. K. Yang, "Solifenacin improves double-J stent-related symptomsin both genders following uncomplicated ureteroscopic lithotripsy," Urolithiasis, vol. 41, pp. 247-252, 2013.

[10] K. Ugur, E. Bilal, T. Murat, F. Gokhan, T. Fatih, and O. Aydin, "Effectiveness of medical treatment in overcoming the ureteral double-J stent related symptoms," Canadian Urological Association Journal, vol. 6, no. 6, pp. 234-237, 2012.

[11] M. Shibasaki, K. Sudoh, O. Inagaki, W. Uchida, and K. Honda, "Effect of the optical isomers of YM-12617 on increased intraurethral pressure induced by phenylephrine in anaesthetized dogs," Journal of Autonomic Pharmacology, vol. 12, no. 4, pp. 263-268, 1992.

[12] M. D. Vardy, H. D. Mitcheson, T.-A. Samuels et al., "Effects of solifenacin on overactive bladder symptoms, symptom bother and other patient-reported outcomes: results from VIBRANTa double-blind, placebo-controlled trial," International Journal of Clinical Practice, vol. 63, no. 12, pp. 1702-1714, 2009.
[13] C.-H. Ho, T.-C. Chang, H.-H. Lin, S.-P. Liu, K.-H. Huang, and H.-J. Yu, "Solifenacin and tolterodine are equally effective in the treatment of overactive bladder symptoms," Journal of the Formosan Medical Association, vol. 109, no. 10, pp. 702-708, 2010.

[14] H. B. Joshi, A. Stainthorpe, R. P. MacDonagh, F. X. Keeley Jr., and A. G. Timoney, "Indwelling ureteral stents: evaluation of symptoms, quality of life and utility," Journal of Urology, vol. 169, no. 3, pp. 1065-1069, 2003.

[15] R. R. Byrne, B. K. Auge, J. Kourambas, R. Munver, F. Delvecchio, and G. M. Preminger, "Routine ureteral stenting is not necessary after ureteroscopy and ureteropyeloscopy: a randomized trial," Journal of Endourology, vol. 16, no. 1, pp. 9-13, 2002.

[16] M. Duvdevani, B. H. Chew, and J. D. Denstedt, "Minimizing symptoms in patients with ureteric stents," Current Opinion in Urology, vol. 16, no. 2, pp. 77-82, 2006.

[17] R. Damiano, R. Autorino, M. de Sio, A. Giacobbe, I. M. Palumbo, and M. D’Armiento, "Effect of tamsulosin in preventing ureteral stent-related morbidity: a prospective study," Journal of Endourology, vol. 22, no. 4, pp. 651-655, 2008.

[18] A. Rane, A. Saleemi, D. Cahill, S. Sriprasad, N. Shrotri, and R. Tiptaft, "Have stent-related symptoms anything to do with placement technique?" Journal of Endourology, vol. 15, no. 7, pp. 741-745, 2001.

[19] D. T. Beiko, B. E. Knudsen, and J. D. Denstedt, "Advances in ureteral stent design," Journal of Endourology, vol. 17, no. 4, pp. 195-199, 2003.

[20] M. D. Dunn, A. J. Portis, S. A. Kahn et al., "Clinical effectiveness of new stent design: randomized single-blind comparison of tail and double-pigtail stents," Journal of Endourology, vol. 14, no. 2, pp. 195-202, 2000.

[21] C.-H. Ho, S.-C. Chen, S.-D. Chung et al., "Determining the appropriate length of a double-pigtail ureteral stent by both stent configurations and related symptoms," Journal of Endourology, vol. 22, no. 7, pp. 1427-1431, 2008.

[22] R. Damiano, R. Autorino, M. de Sio et al., "Does the size of ureteral stent impact urinary symptoms and quality of life? A prospective randomized study," European Urology, vol. 48, no. 4, pp. 673-678, 2005.

[23] B. H. Chew, B. E. Knudsen, L. Nott et al., "Pilot study of ureteral movement in stented patients: first step in understanding dynamic ureteral anatomy to improve stent comfort," Journal of Endourology, vol. 21, no. 9, pp. 1069-1075, 2007.

[24] R. J. Lang, M. E. Davidson, and B. Exintaris, "Pyeloureteral motility and ureteral peristalsis: essential role of sensory nerves and endogenous prostaglandins," Experimental Physiology, vol. 87, no. 2, pp. 129-146, 2002.

[25] C.-J. Wang, S.-W. Huang, and C.-H. Chang, "Effects of tamsulosin on lower urinary tract symptoms due to double-j stent: a prospective study," Urologia Internationalis, vol. 83, no. 1, pp. 66-69, 2009.

[26] A. Agarwal, S. Dhiraaj, V. Singhal, R. Kapoor, and M. Tandon, "Comparison of efficacy of oxybutynin and tolterodine for prevention of catheter related bladder discomfort: a prospective, randomized, placebo-controlled, double-blind study," British Journal of Anaesthesia, vol. 96, no. 3, pp. 377-380, 2006.

[27] K. T. Lim, Y. T. Kim, T. Y. Lee, and S. Y. Park, "Effects of tamsulosin, solifenacin, and combination therapy for the treatment of ureteral stent related discomforts," Korean Journal of Urology, vol. 52, no. 7, pp. 485-488, 2011. 
[28] H. B. Joshi, A. Okeke, N. Newns, F. X. Keeley Jr., and A. G. Timoney, "Characterization of urinary symptoms in patients with ureteral stents," Urology, vol. 59, no. 4, pp. 511-516, 2002.

[29] C. Deliveliotis, M. Chrisofos, E. Gougousis, A. Papatsoris, A. Dellis, and I. M. Varkarakis, "Is there a role for alphal-blockers in treating double-J stent-related symptoms?" Urology, vol. 67, no. 1, pp. 35-39, 2006.

[30] R. Beddingfield, R. N. Pedro, B. Hinck, C. Kreidberg, K. Feia, and M. Monga, "Alfuzosin to relieve ureteral stent discomfort: a prospective, randomized, placebo controlled study," Journal of Urology, vol. 181, no. 1, pp. 170-176, 2009.

[31] U. Kuyumcuoglu, B. Eryildirim, M. Tuncer, G. Faydaci, F. Tarhan, and A. Ozgül, "Effectiveness of medical treatment in overcoming the ureteral double-J stent related symptoms," Canadian Urological Association Journa, vol. 6, no. 6, pp. E234E237, 2012.

[32] R. D. Norris, R. L. Sur, W. P. Springhart et al., "A prospective, randomized, double-blinded placebo-controlled comparison of extended release oxybutynin versus phenazopyridine for the management of postoperative ureteral stent discomfort," Urology, vol. 71, no. 5, pp. 792-795, 2008.

[33] S. C. Park, S. W. Jung, J. W. Lee, and J. S. Rim, "The effects of tolterodine extended release and alfuzosin for the treatment of double-j stent-related symptoms," Journal of Endourology, vol. 23, no. 11, pp. 1913-1917, 2009.

[34] K. T. Lim, Y. T. Kim, T. Y. Lee, and S. Y. Park, "Effects of tamsulosin, solifenacin, and combination therapy for the treatment of ureteral stent related discomforts," Korean Journal of Urology, vol. 52, no. 7, pp. 485-488, 2011.

[35] S. J. Lee, C. Yoo, C. Y. Oh et al., "Stent position is more important than $\alpha$-blockers or anticholinergics for stent-related lower urinary tract symptoms after ureteroscopic ureterolithotomy: a prospective randomized study," Korean Journal of Urology, vol. 51, no. 9, pp. 636-641, 2010. 


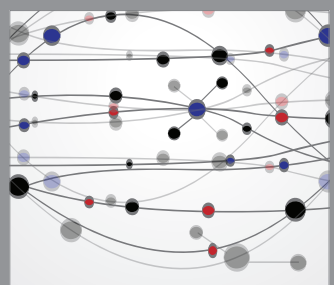

The Scientific World Journal
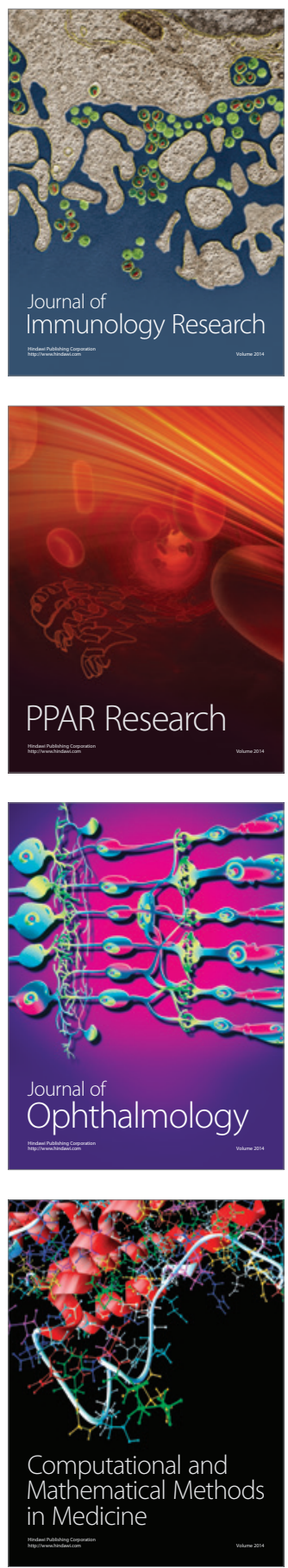



Gastroenterology

Research and Practice


\section{Hindawi}

Submit your manuscripts at

http://www.hindawi.com
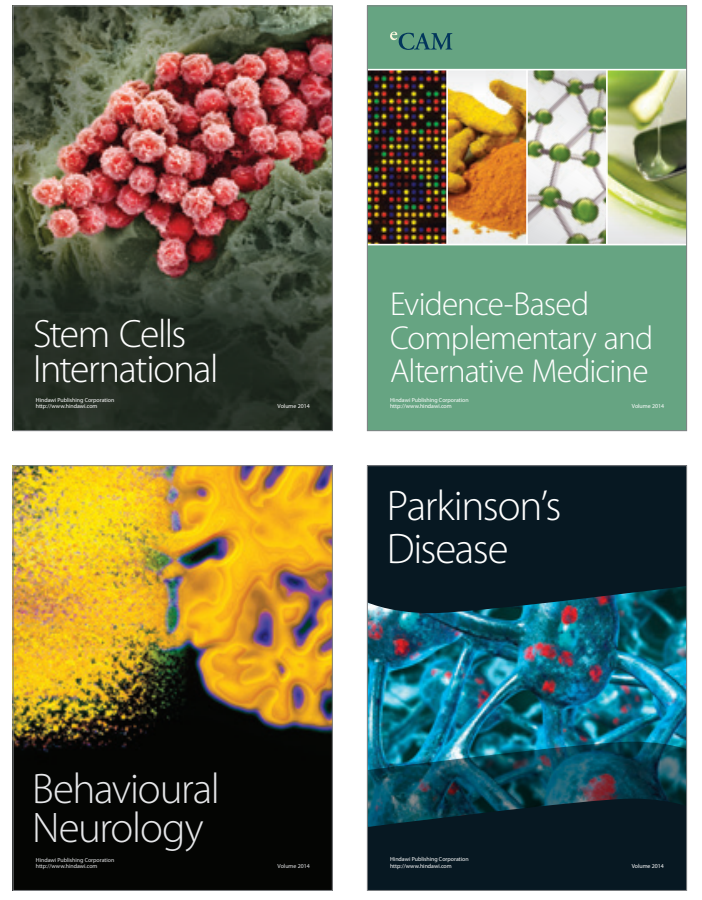
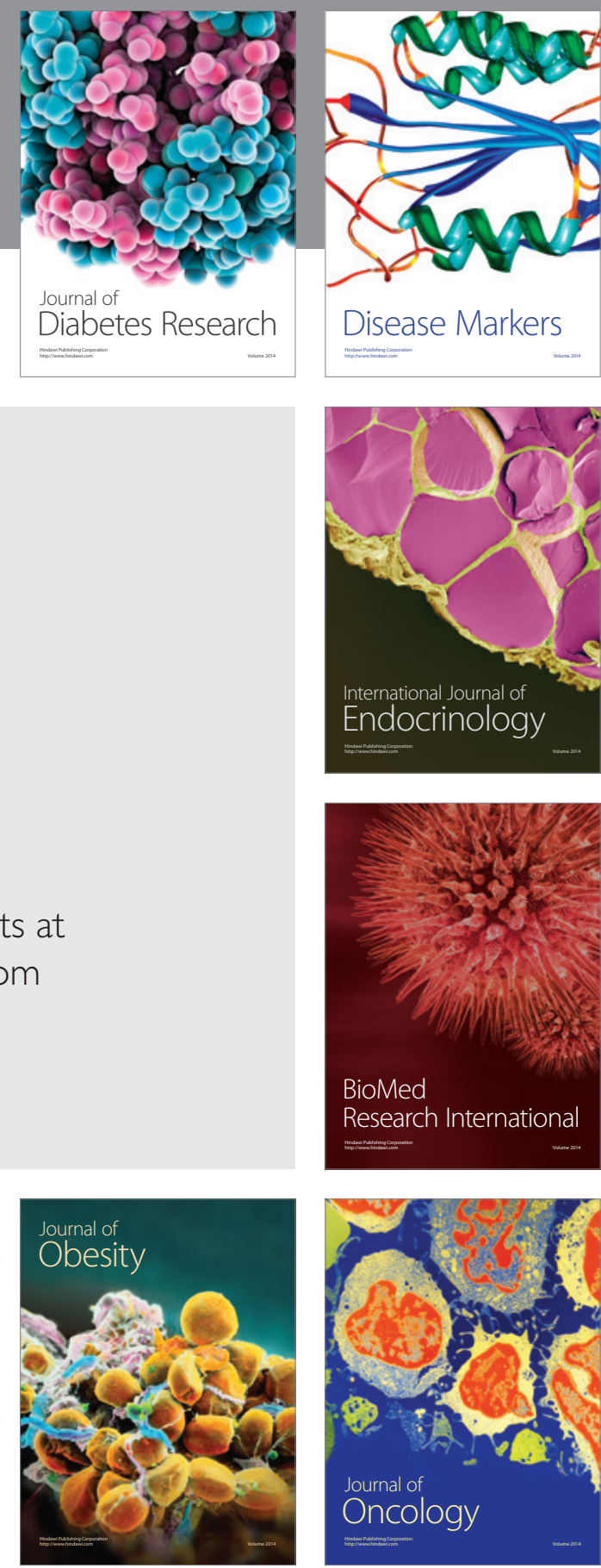

Disease Markers
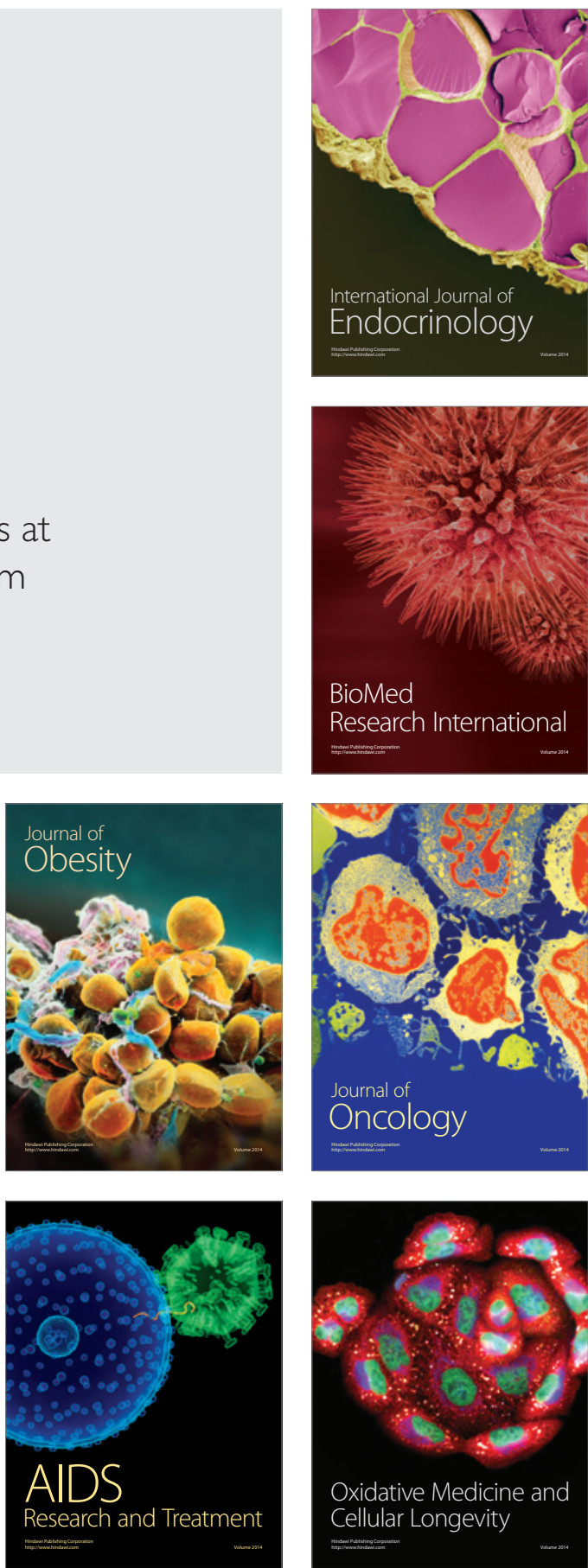